\title{
Mathematical Approach and Implementation of Frequency Mapping Techniques in Power-Line Communications Channel
}

\author{
Tedy Lukusa, Khmaies Ouahada*, Hendrik C. Ferreira \\ Department of Electrical and Electronic Engineering Science, University of Johannesburg, South Africa \\ *Email address for correspondence: kouahada@uj.ac.za \\ Communicated by Giorgio Fotia \\ Received on 05 21, 2017. Accepted on 03 28, 2019.
}

\begin{abstract}
Power-line channel is considered to be a very hostile channel compared to other channels in view of the different types of noise that could exist. Therefore, the choice of the error correcting code and the modulation scheme can play a big role in combating the noise in such a channel. $M$-FSK modulation has shown its robustness for such a type of channel. Two frequency mappings techniques are presented in this paper. In the first technique, $M$ orthogonal frequencies are arranged in sequences based on the value and the position of permutation symbols, while in the second technique, the frequencies are rearranged based on the sign changes of the Walsh-Hadamard transform (WHT). The obtained $M$-FSK modulation is combined to codes based on Viterbi decoding algorithms since Viterbi decoder is considered to be the maximum-likelihood decoding algorithm for convolutional codes and codes with state machine representation. A mathematical approach and implementation of frequency mappings is introduced to investigate the performance of the new designed communication system in the presence of permanent frequency disturbances, also known as narrow-band interference (NBI), such as those encountered in power line communications (PLC) channel.

Keywords: M-FSK Modulation, Power-Line Communications, Viterbi Decoder, Walsh-Hadamard transform, Mapping

AMS subject classification: 94B05,94B10,94B12, 94B35,15B34,42C10
\end{abstract}

\section{Introduction}

The development of digital communications currently progresses at a very rapid pace. High speed communications are operating preferably in fixed connection through fibre optic means. Since this type of communications is quite costly to implement even in developed countries, the power-line infrastructure - through which every household is connected to the power grid at low voltages - could be used as a communication medium since it uses an existing infrastructure.

The power-line communications (PLC) channel [1] is considered as a hostile channel and to overcome the noise in this type of channel, Vinck et al. [2] have introduced the concept of coded modulation for power-line communications. Specifically, they have combined $M$-FSK with permutation codes to make the transmissions robust against permanent frequency disturbances, impulse noise and also background noise.

We can consider the designed permutation trellis codes (PTC) [3] based on the use of distancepreserving mappings which are coding techniques that map the outputs of a convolutional code to other codewords from a permutation codebook of less error-correction capabilities.

The second technique used in this paper is the mapping of frequencies onto the outputs of convolutional codes for use in power line communications. We investigate the performance of the combination of the Walsh-Hadamard transform [4], the frequency sequence from $M$-FSK modulation scheme and the outputs of convolutional codes in AWGN channel and in the presence of narrow-band noise [5].

Walsh-Hadamard transform is considered as the simplest of the usual orthogonal transform like the Fourier transform. Walsh-Hadamard matrices have been used successfully in different applications such as switching networks [6], error-correcting codes and signal processing $[7,8]$. 
The idea of making use of $M$-FSK modulation and non-coherent detection in a diversity scheme, which conforms to the European Committee for Electrotechnical Standardization Norms (CENELEC) [9], EN 50065.1, part 6.3.2, is to make transmissions robust against Narrow-Band-, Broad-Band and background noise disturbances in such hostile channel [10].

The approved IEEE 1901.2 standard for low frequency (less than $500 \mathrm{kHz}$ ) Narrow-Band power line communications for smart grid applications has raised the bar of performance with added enhancements for increased data rates greater than $300 \mathrm{kbps}$. Also the standards for the physical and medium access control layer G3-PLC and PRIME have shown their good performance in the CENELEC A band by cyclic prefix (CP) Orthogonal Frequency Division Multiplexing (OFDM) in combination with coded Differential Phase Shift Keying (DPSK) and certain error correction codes like Reed-Solomon and convolutional codes.

Our research is motivated by the CENELEC regulations for power-line communications in the low frequency region. These regulations require a maximum modulated signal amplitude and thus reduce the number of modulation schemes that can be used. OFDM has the disadvantage of a non constant envelope and thus special peak reduction techniques have to be applied. Our approach is to use a constant envelope modulated signal and use coding to combat the noisy environment.

The paper is organized as follows. In Section 2 a brief description of the power line communications channel and its proposed model were presented as well an introduction to the $M$-FSK modulation scheme used in this paper. In Section 3 we present a mathematical approach of the frequency mappings used in this paper from permutation sequences to frequencies and from sign changes property in the WalshHadamard transform to frequencies. The communication system is explained in Section 4. Finally a conclusion on the performance of frequency mapping techniques and the mathematical approach and implementation results are presented in Section 5.

\section{Communications System Design}

\subsection{Power-Line Communications Channel}

Power-line communications channel has three main different types of noise [10] presented as follow:

- The background noise, which is equivalent to additive white Gaussian noise (AWGN), is usually caused by natural sources like the radiation from the sun.

- The impulse noise, which can be presented for a short duration and can affect many or all frequencies in the same time slot. This type of noise is usually caused by switching equipment or sometimes lightning's strikes.

- The permanent frequency disturbances or narrow-band noise can affect some frequencies and can also be present over a period of time. The source of this type of noise could be from television sets or computer terminals.

In this paper and due to the importance if this kind of noise, we limit our investigation to narrowband interference (NBI). In the case of narrowband noise, which is described differently to the AWGN channel [10], its source has a probability $\boldsymbol{p}_{n}$ to be present for a duration of $T_{n}$ frequency-time slot or its corresponding number of sign-changes, where $T_{n} \in[0, \infty)$. We assume that the narrowband noise signal has an energy $E_{n s}$ which is much higher than the energy of the transmitted signal and with a frequency $f_{n s}=f_{i}$, where $f_{i}$ corresponds to one of the transmission frequencies. The noise signal with energy, $E_{n s}$, is then added to the transmitted symbol energy which is already corrupted by the AWGN, $n_{i}$. This will cause a total saturation of the signal at the corresponding frequency. It is important to mention that the phase difference between the noise and the transmitted signal will contribute to the change of the resultant energy of the transmitted symbols. Since we are interested in this work only in the technique of correcting transmitted data in a narrowband channel, we will not take into consideration the effect of the phase difference on the energy of the transmitted symbol.

Each frequency is presented at a specific time domain. If we have $M$ orthogonal frequencies representing signals transmitted into the channel, the mathematical presentation of these $M$ orthogonal 
frequencies in the time domain can be in the form of a matrix $\mathcal{F}$ as follows [11].

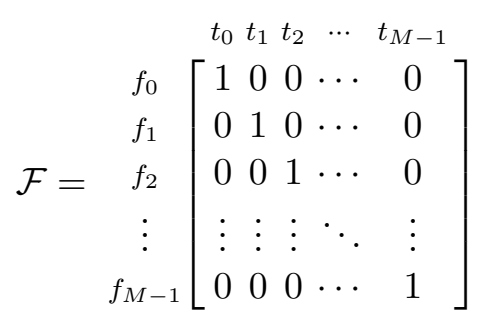

We allocate one to each frequency at its corresponding time slot following the order of it appearance in the frequency signal sequences. For any type of disturbances mentioned in the introduction, the location of ones will differ from the normal and general repartition as depicted in the matrix $\mathcal{F}$. Example 6.11 in the Appendix explains the construction of $\mathcal{F}$ for $M=8$ and also the its presentation for the noise in the PLC channel.

It is noted that narrow-band noise interferes at certain specific frequencies can cause a total saturation of the signal at the corresponding frequency and will result of the appearance of that specific frequency for a longer period of time. This phenomenon can be seen in the frequency-time presentation of the transmitted frequencies or the matrix $\mathcal{F}$, where ones occupy the whole row at the specific effected frequency by the noise as depicted in Example 6.1 in the Appendix.

\subsection{M-ary FSK Modulation}

The discussion in this section revolves around a few of the major properties of the $M$-ary frequency shift keying (FSK) modulation scheme [12] that has been used in the telecommunication systems, especially in power-line communications. Combined with other codes like permutation codes, $M$-ary FSK has shown robustness against permanent frequency disturbances and impulse noise [10]. The modulation scheme with its constant envelope signal is in agreement with CENELEC.

\subsubsection{Non-coherent Detection of Orthogonal Signals with M-ary FSK Modulation}

$M$-ary FSK is considered to be an orthogonal frequency modulation scheme, same as OFDM modulation. In this communication system we consider using $M$ orthogonal waveforms to transmit information, presented as follows:

$$
s_{1}(t), s_{2}(t), \ldots, s_{M}(t) .
$$

The signal space has a dimension $M$ and the received vector $r$ is given by

$$
\begin{aligned}
r & =\left[r_{1}, \ldots, r_{M}\right], \quad \text { with } \\
r_{i} & = \begin{cases}\sqrt{E_{s}} e^{j \phi}+n_{i, c}+j n_{i, s}, & \text { for } i=k, \\
n_{i, c}+j n_{i, s}, & \text { for } i \neq k,\end{cases} \\
p\left(r \mid s_{k}\right) & =\left(\frac{1}{2 \pi \sigma_{v}^{2}}\right)^{N} e^{\left(|r|^{2}+E_{s}^{2}\right) / 2 \sigma_{v}^{2}} I_{0}\left(\frac{\sqrt{E_{s}\left|r_{k}\right|^{2}}}{\sigma_{v}^{2}}\right),
\end{aligned}
$$

where, $E_{s}$ represents the energy per symbol, $\phi$, the phase shift of the signal and $I_{0}$ is the modified Bessel function of order zero.

At the demodulator the optimum detector computes the magnitude of different coordinates of the received vector and chooses the maximum as depicted in the following expression,

$$
\max \left\{\left|r_{1}\right|^{2},\left|r_{2}\right|^{2}, \ldots,\left|r_{M}\right|^{2}\right\}
$$


The combination of $M$-FSK modulation and coding for a constant envelope modulation signal has the advantage of having a frequency spreading that can help us avoiding some bad parts of the frequency spectrum to facilitate correction of frequency disturbances and impulse noise simultaneously. $M$-FSK has the advantage of a constant envelope signal modulation and a demodulation in a coherent as also in a non-coherent way. As it is known, in a $M$-FSK modulation scheme, symbols are modulated as one of the sinusoidal waves described by the following description [10],

$$
s_{i}(t)=\sqrt{\frac{2 E_{s}}{T_{s}} \cos \left(2 \pi f_{i} t\right)} ; 0 \leq t \leq T_{s},
$$

taking $1 \leq i \leq M$ and $E_{s}$ as the signal energy per modulation symbol and $f_{i}=f_{0}+\frac{i-1}{T_{s}}, 1 \leq i \leq M$.

The correspondence between symbols generated by the distance preserving mappings (DPMs) and the frequencies from the $M$-FSK modulation make this combination very practical in a environment like the power-line communications channel.

\subsubsection{Demodulation}

Figure 1 shows a detailed $M$-FSK demodulator, where we have $2 M$ correlators, as there are two for each possible transmitted frequency. The receiver for non-coherent demodulation does not have to know the phase. The simplest form of non-coherent demodulation is envelope detection, where the demodulator computes $M$ envelopes and chooses the largest envelope as an estimated output for the transmitted frequency.

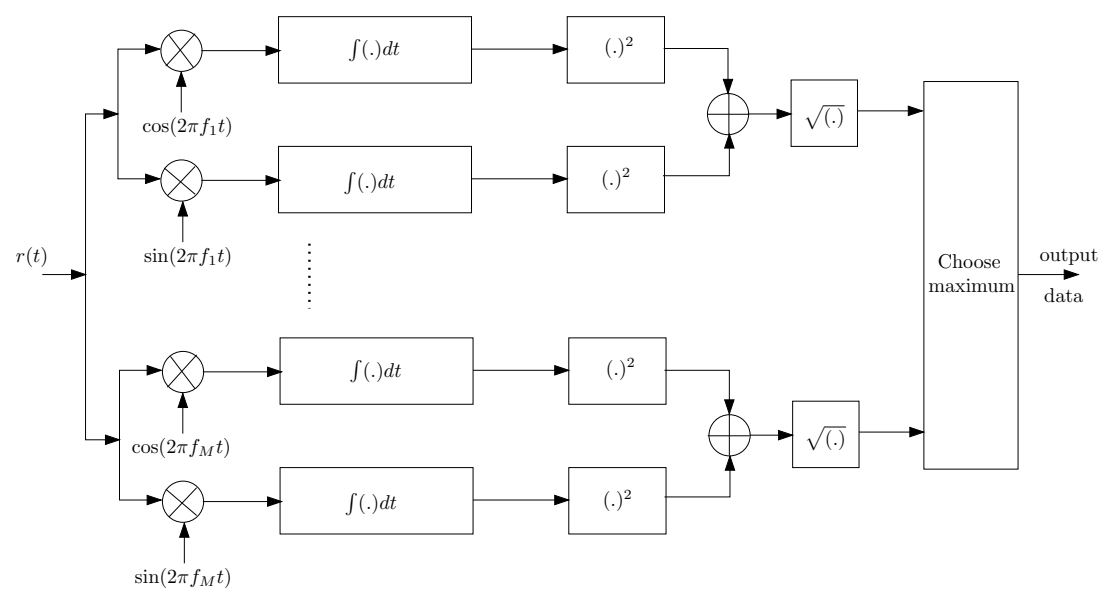

Figure 1. Non-coherent $M$-FSK demodulator

Since the $M$-FSK modulation scheme is combined with codes to combat certain types of noise in the power-line communication channel under investigation, we present here the definitions of the three types of detector/decoder combinations of threshold detection [10] that we have used in our work. We use for the purpose of simplification the matrix $M$ by $M$, where the rows represent the number of frequencies used in the $M$-FSK modulation scheme, and the columns represent the length of the codeword of the permutation sequence.

Definition 2.1. Envelope Detector (ED) computes the largest value of the envelope of the signal, we allocate the value 1 to the corresponding element in the matrix $\mathcal{F}$. Hence it is assumed the corresponding frequency has been transmitted.

Definition 2.2. In this type of threshold detector (TD), we refine our decision and the optimization depends on the energy $E_{s}$ and $\sigma_{v}^{2}$. We usually choose the value of $\tau \times \sqrt{E_{s}}$ and for practical reasons we can choose $0.6 \leq \tau \leq 0.9$.

Definition 2.3. Viterbi [13] proposed a threshold called ratio threshold test (VRTT) for mitigating the partial-band or multitone interferences when invoked as an erasure insertion scheme. 


\section{Mathematical Approach for Frequency Mappings}

\subsection{Permutation Symbols and Frequency Mappings}

Using the technique of distance-preserving mappings, the output of a convolutional encoder can be mapped to permutation sequences, creating a permutation trellis code (PTC) [14]. The purpose behind this mapping is to firstly obtain suitably constrained output code sequences and secondly to exploit the error correction characteristics of the new code with the use of the Viterbi algorithm [14].

Let $\mathcal{P}_{M}=\{1,2, \ldots, M\}$, an integer sequence of length $M$ and let $\mathcal{S}_{M}$ denote the set of all permutations of symbols in $\mathcal{P}_{M} . \mathbb{Z}_{2}^{n}$ is the set of all binary vectors of length $n$.

The mappings considered, take all possible binary sequences of length $n$, from the binary set denoted by $\mathcal{C}_{b}$, with $\left|\mathcal{C}_{b}\right|=2^{n}$, to a subset of $\mathcal{S}_{M}$, denoted by $\mathcal{C}_{p}$ where we have $\left|\mathcal{C}_{p}\right|=\left|\mathcal{C}_{b}\right|$.

In [3] it was shown how the output binary $n$-tuple code symbols from an $R=m / n$ convolutional code could be mapped to non-binary $M$-tuple permutation code symbols as depicted in Figure 2 to create a permutation trellis code.

Explaining the mapping technique in a simple way, we make use of the convolutional code with rate $R=1 / 2$ and constraint length $K=3$ [13] as a base code. The output of the encoder, which is a set of binary 2 -tuple code symbols, can be mapped to a set of permutation $M$-tuples. The corresponding state systems appear in Figure 3.

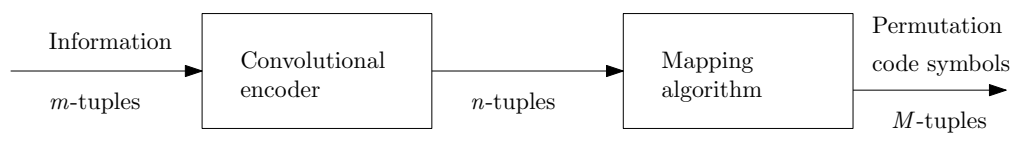

Figure 2. Encoding process for distance-preserving mapping codes

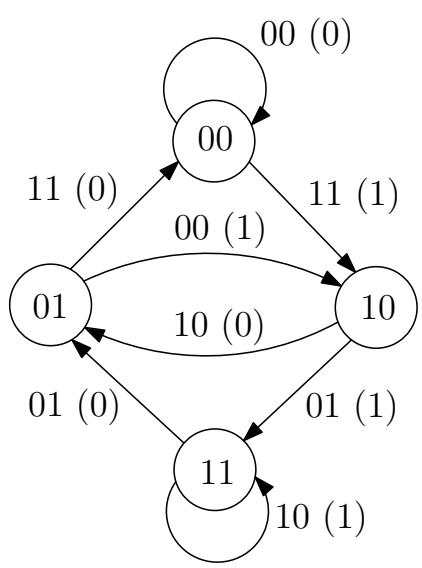

(a)

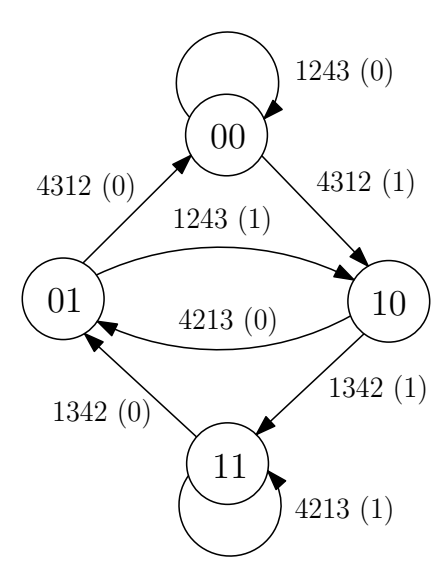

(b)

Figure 3. State systems for (a) convolutional base code and (b) permutation trellis code

After mapping binary sequences onto permutation sequences and after choosing non-coherent $M$-FSK modulation, the corresponding frequency mapping as in the case of $M=4$, is depicted in the following mapping:

$$
\left\{\begin{array}{ll}
0 & 0 \\
0 & 1 \\
1 & 0 \\
1 & 1
\end{array}\right\} \rightarrow\left\{\begin{array}{llll}
1 & 2 & 4 & 3 \\
1 & 3 & 4 & 2 \\
4 & 2 & 1 & 3 \\
4 & 3 & 1 & 2
\end{array}\right\} \rightarrow\left\{\begin{array}{llll}
f_{1} & f_{2} & f_{4} & f_{3} \\
f_{1} & f_{3} & f_{4} & f_{2} \\
f_{4} & f_{2} & f_{1} & f_{3} \\
f_{4} & f_{3} & f_{1} & f_{2}
\end{array}\right\}
$$

The strength of error correction capability of the resultant permutation trellis code is based on the value of the Hamming distance defined as follows: 
Definition 3.1. The Hamming distance $d_{H}\left(\mathbf{x}_{i}, \mathbf{x}_{j}\right)$ is defined as the number of positions in which the two sequences $\mathbf{x}_{i}$ and $\mathbf{x}_{j}$ differ.

Applying the definition of the Hamming distance to binary and non-binary sequences, we can denote by $\mathbf{D}=\left[d_{i j}\right]$ the distance matrix whose entries are the Hamming distances between two binary sequences $\mathbf{x}_{i}$ and $\mathbf{x}_{j}$ defined as follows:

$$
\mathbf{D}=\left[d_{i j}\right] \text { with } d_{i j}=d_{H}\left(\mathbf{x}_{i}, \mathbf{x}_{j}\right)
$$

Similarly for permutation sequences we denote by $\mathbf{E}=\left[e_{i j}\right]$ the distance matrix whose entries are the Hamming distances between two permutation sequences $\mathbf{y}_{i}$ and $\mathbf{y}_{j}$ defined as follows:

$$
\mathbf{E}=\left[e_{i j}\right] \text { with } e_{i j}=d_{H}\left(\mathbf{y}_{i}, \mathbf{y}_{j}\right) .
$$

The sum of all the distances in $\mathbf{D}$ and $\mathbf{E}$ are defined as follow:

$$
\begin{gathered}
\|\mathbf{D}\|=\sum_{i=1}^{\left|\mathcal{C}_{b}\right|} \sum_{j=1}^{\left|\mathcal{C}_{b}\right|} d_{i j} . \\
\|\mathbf{E}\|=\sum_{i=1}^{\left|\mathcal{C}_{p}\right|} \sum_{j=1}^{\left|\mathcal{C}_{p}\right|} e_{i j} .
\end{gathered}
$$

The total distances, $\|\mathbf{D}\|$ and $\|\mathbf{E}\|$ play a role in the permutation code's error correcting capabilities, as was shown in [15]. In the case of $M=4$ as depicted in Example 6.2 in the Appendix, the mapping can increase the distance and therefore the error correction capability of the resultant code.

In general, three types of DPMs can be obtained, depending on how the Hamming distance is preserved as depicted in the following definitions.

Definition 3.2. Distance-increasing mappings (DIMs), where $e_{i j} \geq d_{i j}+\delta, \delta \in\{1,2, \ldots\}, \forall i \neq j$.

Definition 3.3. Distance-conserving mappings (DCMs), where $e_{i j} \geq d_{i j}, \forall i \neq j$ and equality achieved at least once.

Definition 3.4. Distance-reducing mappings (DRMs), where $e_{i j} \geq d_{i j}+\delta, \delta \in\{-1,-2, \ldots\}, \forall i \neq j$.

Any DPMs construction is represented by a set $(M, n, \delta)$, where $M$ represents the length of the permutation sequence, $n$ is the length of the convolutional code output and $\delta$ gives an idea about the type of the mapping.

Example 6.3 in the Appendix shows in details the general flow chart of the mapping process and also the algorithm of distance conserving mapping (DCM) for $M=n=4$ using the cube construction [15] as an example of an optimum construction for the design of permutation trellis codes.

\subsection{Walsh-Hadamard Transform and Frequency Mappings}

As presented in the literature [4], Walsh-Hadamard transform (WHT) consists of a projection onto a set of square waves called Walsh functions. The WHT coefficients are called sequence components and the Walsh functions are ordered by the number of their zero-crossings.

The Walsh-Hadamard matrix $H_{1}$ is defined as:

$$
H_{1}=\frac{1}{\sqrt{2}}\left[\begin{array}{l}
+1+1 \\
+1-1
\end{array}\right],
$$


and by using the Kronecker product of two matrices, the Walsh-Hadamard matrix $H_{n}$ of order $2^{n}$ is defined recursively by:

$$
H_{n}=H_{1} \otimes H_{n-1}=\left[\begin{array}{c}
+H_{n-1}+H_{n-1} \\
+H_{n-1}-H_{n-1}
\end{array}\right] .
$$

Example 6.4 in the Appendix shows the Walsh-Hadamard matrices for the cases of $n=2$ and $n=3$. The rows in a Walsh-Hadamard matrix represent the Walsh functions, which are defined as follow:

$$
W(k, t)=\prod_{i=0}^{r-1} \operatorname{Sign}\left[\cos ^{k_{i}}\left(2^{i} \pi\right) t\right]
$$

where $r=\left\lfloor\left(\log _{2}(k)+1\right)\right\rfloor, k$ is the index of Walsh function, $k_{i}$ is the $i^{t h}$ bit of $k$ in binary.

If we consider that Walsh-functions consist of trains of square pulses, then we can introduce the concept of sign change in each Walsh function [7]. The number of sign changes in a row of a WalshHadamard matrix is called sequency.

As it is known that Walsh-Hadamard matrix is constructed from the Hadamard matrix itself by just rearranging the order of the rows of the matrix taking into consideration the number of sign changes in each corresponding Walsh functions. Since we are only interested in the number of sign changes, we will focus in the rest of the paper on the Hadamard matrices.

Since there is a strong link between the number of sign changes in each row of a Hadamard matrix and the corresponding frequency, we can consider a proportionality between the value of the frequency and the number of sign changes in each row of the Hadamard matrix [7]. Usually the first Hadamard row has no sign changes, this will lead to a frequency null which can be presented as a DC signal. In practical the proportionality is in fact a mean to monitor the variation of the frequencies values. Therefore we consider the Hadamard first row has a corresponding frequency with the lowest value and as much as the number of sign changes increase, the corresponding frequency value is increasing. This relationship between the number of sign changes and the frequency value is used to map the transmitted information data as it will be explained in the following sections. Finally we can say that there is a strong link between the sign changes of a Hadamard transform, the frequencies of the transmitted signals and the $M$-FSK modulation scheme.

$$
\begin{aligned}
& \text { Hadamard Transform } \\
& {\left[\begin{array}{cccc}
+1 & +1 & +1 & +1 \\
+1 & -1 & +1 & -1 \\
+1 & +1 & -1 & -1 \\
+1 & -1 & -1 & +1
\end{array}\right] \longrightarrow\left[\begin{array}{c}
0 \\
3 \\
1 \\
2
\end{array}\right]}
\end{aligned}
$$

Figure 4. Walsh-Hadamard matrix and sign changes: $M=4$

For the case of $n=2$, we have four rows in our Hadamard matrix as presented in Figure 4. It is clear from the sequency represented by numbers in the column presented and in the form of a set $\{0,3,1,2\}$, are actually the numbers of zero-crossings or sign changes in each row.

The sequency is similar at certain limit to, but different from, frequency in the sense that it measures the rate of change of non-periodical signals.

A very good advantage of using Hadamard matrix is that the dimension of this matrix is actually $2^{n} \times 2^{n}$, which matches with number of frequencies used in $M$-FSK as $M=2^{n}$.

From another angle, we can make an approach between a sign change and a data shift. As it can be seen in Figure 5, changing a sign of a data is the same as shifting its corresponding binary sequence using the one's complement rule. 


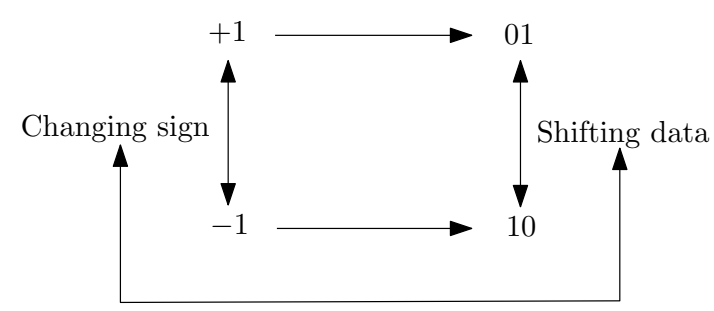

Figure 5. Sign change and data shift approach

Taking into consideration the proportionality between the frequency value and the number of sign changes, we can admit that increasing the number of sign changes will lead to an increase in the frequency values. As an example, in practical using a scope, if we take a sine wave signal and then increase its frequency value, we can see that the signal wave is shifting to the left. This confirm with our approach as explained in Figure 5.

linking more the relationship between Figures 4 and 5, we can say that the obtained set $\{0,3,1,2\}$ represents also the number of shifts, the $M$ frequencies can make to obtained the optimum sequence for mapping. if we take the first symbol, 0, which means no shifts then the obtained set of frequencies is $\left\{f_{0}, f_{3}, f_{1}, f_{2}\right\}$. in the case of 3 and starting with frequency $f_{3}$, the obtained sequence is obtained after three shifts, which will correspond to $\left\{f_{3}, f_{0}, f_{1}, f_{2}\right\}$ as depicted in Figure 20 in Example 6.4 in the Appendix.

From what is presented above about the relationship between sign changes, frequency increase and data shifting, we can present the main idea in this paper as follows. The Hadamard transform will provide us with the sequency, which will lead us to the corresponding frequencies. Then we have to shift these frequencies to obtain the corresponding frequency sequences for the corresponding $M$-FSK modulation scheme. Example 6.1 in the Appendix, where $n=2$, shows in details the progress from Hadamard transform to the frequency mappings based on the sign changes.

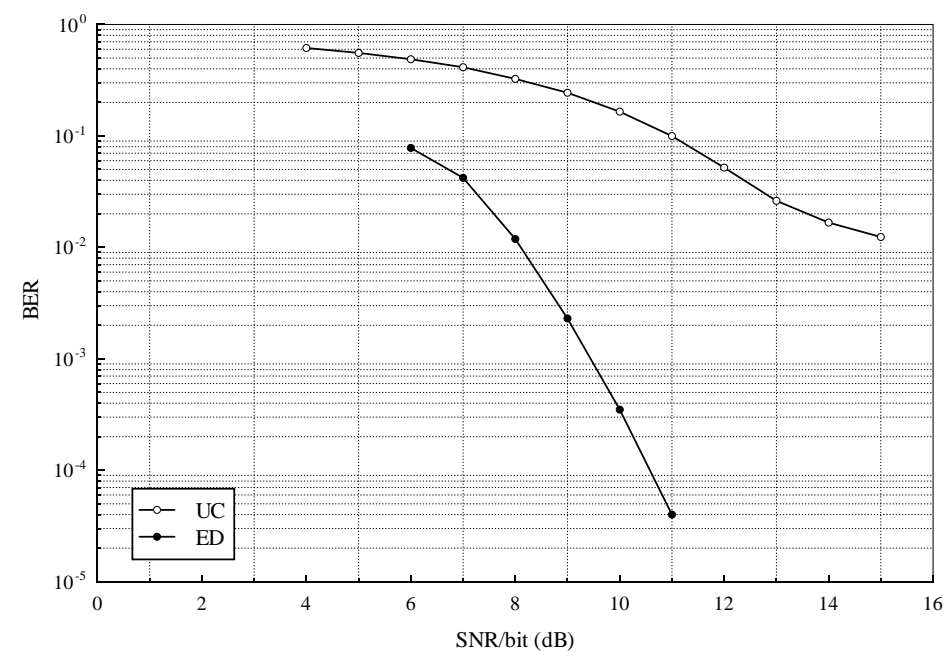

Figure 6. Performance of combined PTC and 8-FSK with ED detector in the presence of NBI

\section{Mathematical Implementation of Frequency Mappings}

\subsection{Permutation Sequences}

In this section, we make use of different detectors that $M$-FSK modulation can use and see the performance of our permutation trellis codes when Narrow-Band noise [10] is present. 


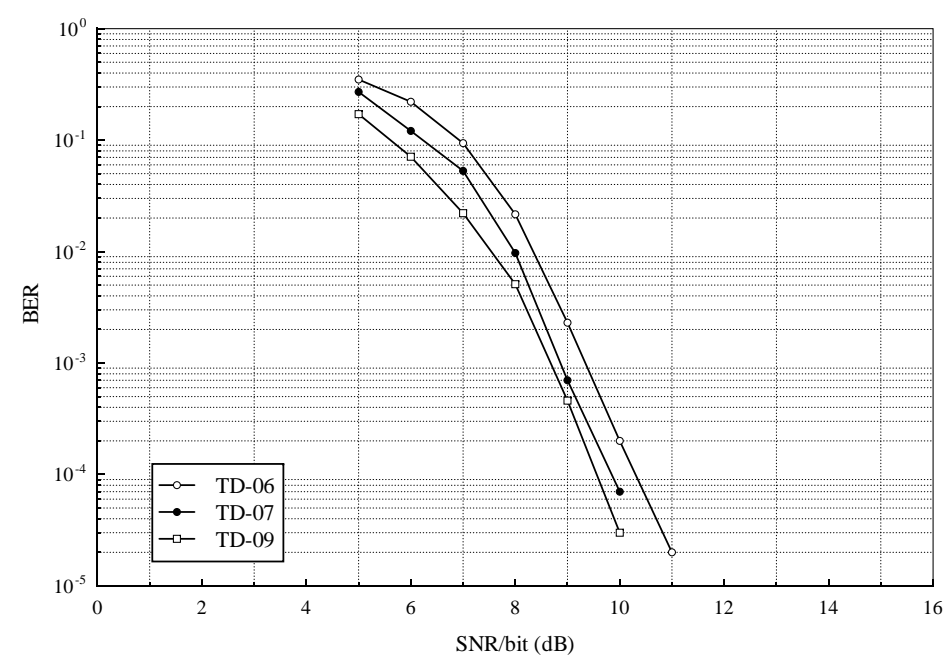

Figure 7. Performance of combined PTC and 8-FSK with TD detector in the presence of NBI

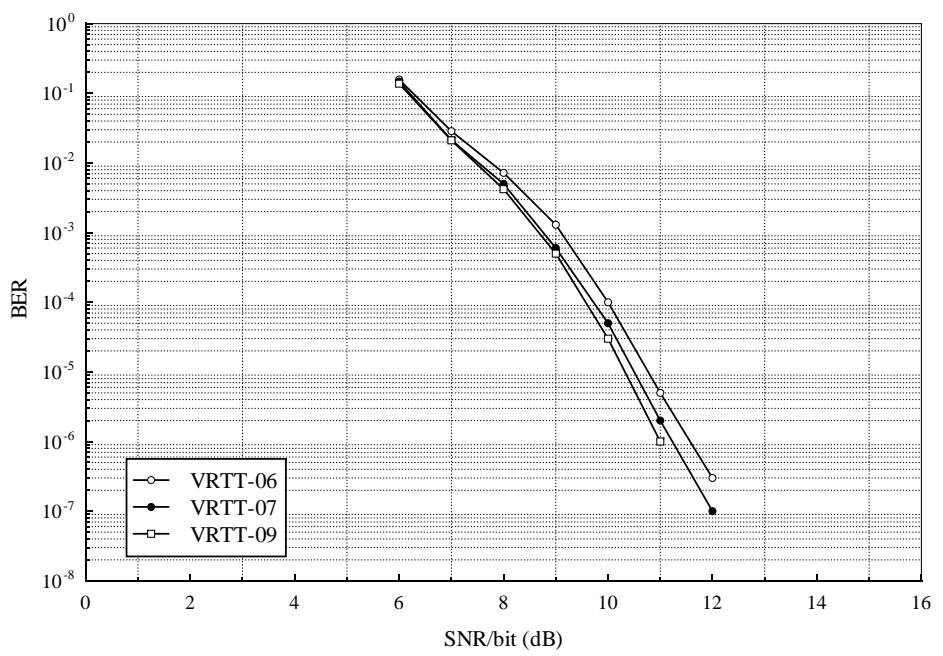

Figure 8. Performance of combined PTC and 8-FSK with VRTT detector in the presence of NBI

As discussed previously, when combining PTC codes with an $M$-FSK modulator, every symbol, $1,2, \ldots, M$ corresponds uniquely to one of the $M$ frequencies. The $M$-ary symbols are transmitted in time as the corresponding frequencies, thus the transmitted signal has a constant envelope.

In the case of $M=8$ and to transmit a permutation sequence as $\{1,3,4,2,6,5,8,7\}$, the corresponding frequencies to each symbol are $\left\{f_{1}, f_{3}, f_{4}, f_{2}, f_{6}, f_{5}, f_{8}, f_{7}\right\}$ respectively. These frequencies sent over the channel will represent our 8-FSK frequencies mappings as it was the case for $M=4$ presented in Equation (2).

We run our simulation for uncoded data (UC) and our PTC codes for different detectors as the envelope detector (ED), the threshold detector (TD) for different values of its threshold $\tau$, and also for the Viterbi's ratio threshold detector (VRTT) for different values of $\lambda$. Although TD and VRTT detector are designed for erasure channels, we make use of them of their soft decision properties and consider our channel as not an erasure channel. The obtained results for the ED detector, the TD detector and the VRTT detector are respectively presented in Figures 6, 7 and 8. 


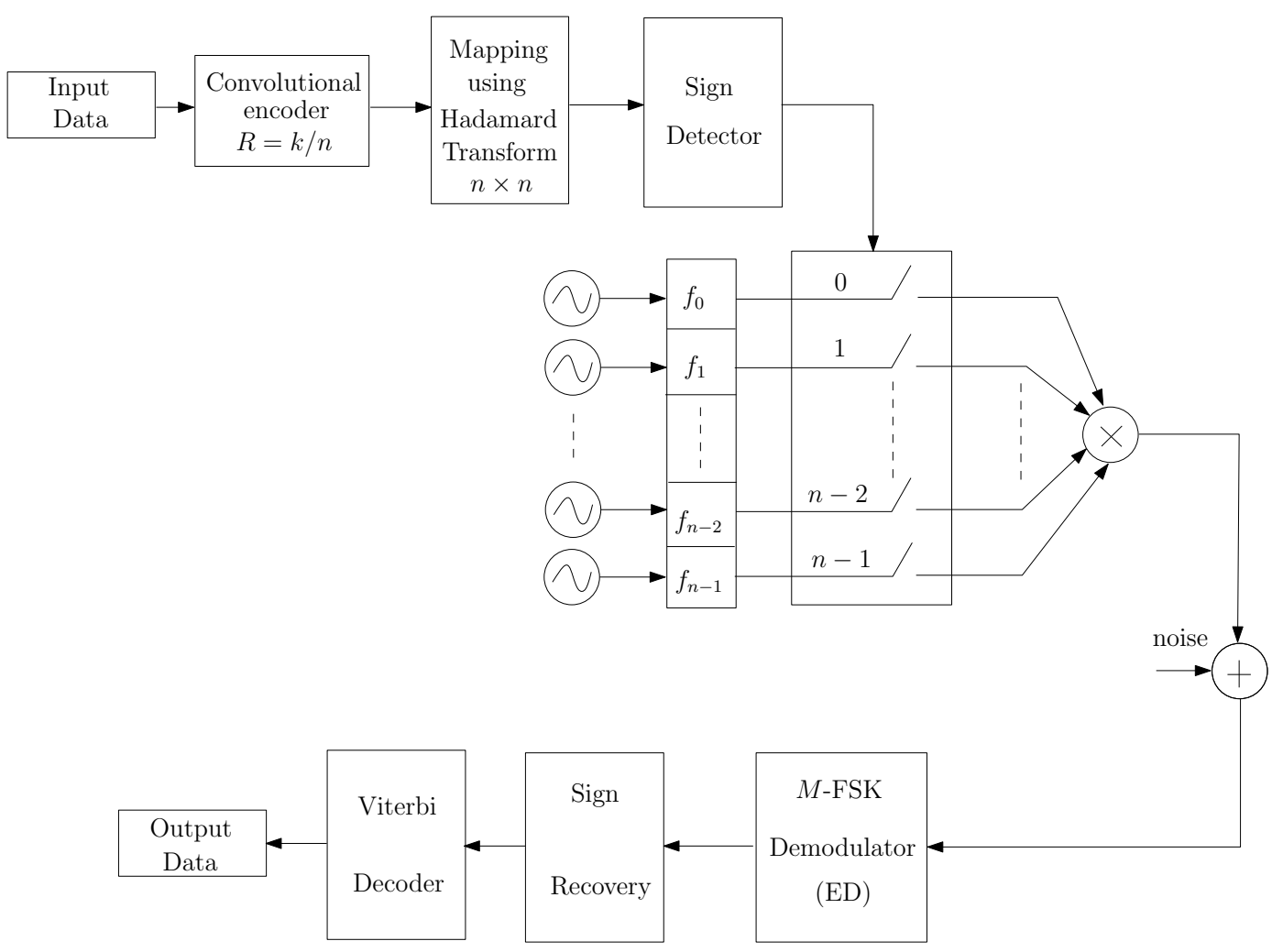

Figure 9. Communication System using Hadamard Transform

\subsection{Walsh-Hadamard Sequences}

\subsubsection{Communication System}

Since we have to get benefit of the Viterbi decoding algorithm, we use our transmitted data as the outputs of a convolutional code with rate and constraint length chosen in a way that suits the corresponding Hadamard matrix dimensions.

The major steps in our proposed communication system presented in Figure 9 are as follow:

- Hadamard mapping

- Sign detector

- Multiplexer

- Sign Recovery

- Viterbi decoder

It is clear from Figure 9, that the convolutional code outputs are mapped onto the rows of the Hadamard matrix, and this has to do with the Walsh functions that contains the sign changes. From each row we can detect the corresponding sign using a sign detector based on Walsh functions presented previously. This will play a key role in the control of switches that allow signals, generated from different signal generators, with different frequencies and which correspond to the number of sign changes to be transmitted. The switches are sequential and work in an increasing order that start from the corresponding frequency of the detected sign and followed by other $M-1$ frequencies. All these frequencies are multiplexed and their corresponding signals are transmitted through the channel.

After the signals were transmitted through the channel and exposed to the noise, the demodulator with envelope detector is used to demodulate them.

Each frequency has a value and since we have a proportionality between these values and the number of sign changes, we can generate again the corresponding sign change to each frequency. If we look at the frequency mapping at the beginning of this paper, we can say that the number of sign changes is 
actually the index of each start frequency. Therefore, generating the number of sign changes is the same as generating permutation sequences.

After generating all the information presented in a permutation sequences form, we can consider these sequences as they were mapped onto the outputs of the convolutional code and thus the Viterbi decoding algorithm will be used easily.

\subsubsection{Frequency Mappings and Viterbi Decoding}

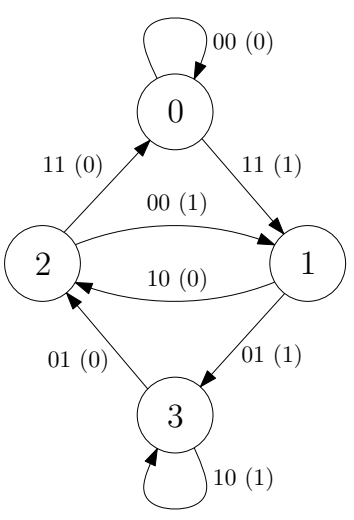

(a)

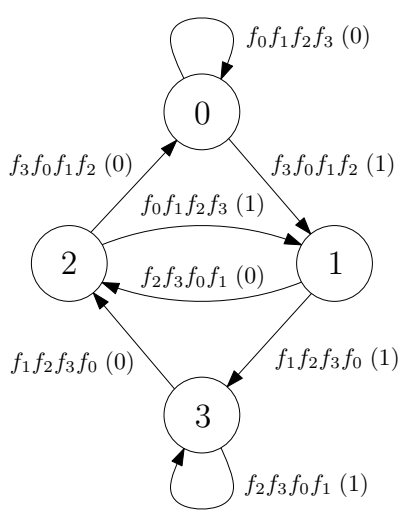

(b)

Figure 10. Frequency mappings

The technique of mapping is usually introduced as the mapping of permutation sequences onto the convolutional outputs. This type of mappings require a construction [15], which is based on certain algorithm to choose the right set of permutation sequences that can give better performance in the error correction process.

In our case, we managed to eliminated the step of a construction and replace it with the Hadamard transform that has the properties of selecting the right frequency sequences of the signal that will be transmitted into the channel.

Figure 10 shows the replacement made for each corresponding convolutional output with the corresponding frequency sequences as the results of the Hadamard transform mappings that was explained in the communication systems presented in Figure 9.

\subsubsection{Simulation Setup}

As it was depicted in Figure 9, and after the demodulation, the number of signs or sign changes will be recovered from the corresponding frequencies' values of the demodulated signals. As the relationship between frequencies and number of sign changes still stand true, we can recover the sequences of the number of sign changes or their corresponding permutation sequences.

The obtained sequences will be used in calculating the distances in each branch of the trellis diagram of the corresponding convolutional code. The calculation of distances in each branch is actually the results of the comparison between the expected frequency sequences and the received ones. Since each expected frequency sequence corresponds to an $M \times M$ matrix denoted by $\mathcal{F}$ and each received frequency matrix corresponds as well to an $M \times M$ matrix denoted by $\mathcal{F}^{\prime}$, the distance for each branch will be calculated as follows:

$$
d_{\text {branch }}=\sum_{1 \leq i, j \leq M}\left(\mathcal{F}_{i j} \oplus \mathcal{F}_{i j}^{\prime}\right),
$$

where $d_{\text {branch }}$ represents the branch metric, $\mathcal{F}_{i j}$ are the entries in the branch expected frequency matrix, $\mathcal{F}_{i j}^{\prime}$ are the entries in the received frequency matrix and the operator $\oplus$, represents the binary XOR function. 
If we break down the matrices $\mathcal{F}$ or $\mathcal{F}^{\prime}$ into row and make use of the XOR function, the calculation of metrics using Equation (10) will be similar to the calculation of metrics using the Hamming distances.

\subsubsection{Simulation Results}

We have used a convolutional code with rate $R=1 / 2$ and constraint length $K=3$ as our source of information. The state machine presented in Figure 10(b), shows the number of states and the number of frequencies outputs for their corresponding inputs, in this case we have $M=4$.

We run our simulations for different values of length and duration of NBI, $T_{n}$, which are usually for the sake of simplification, chosen to be multiple of $M$, the number of frequencies of the transmitted signals.

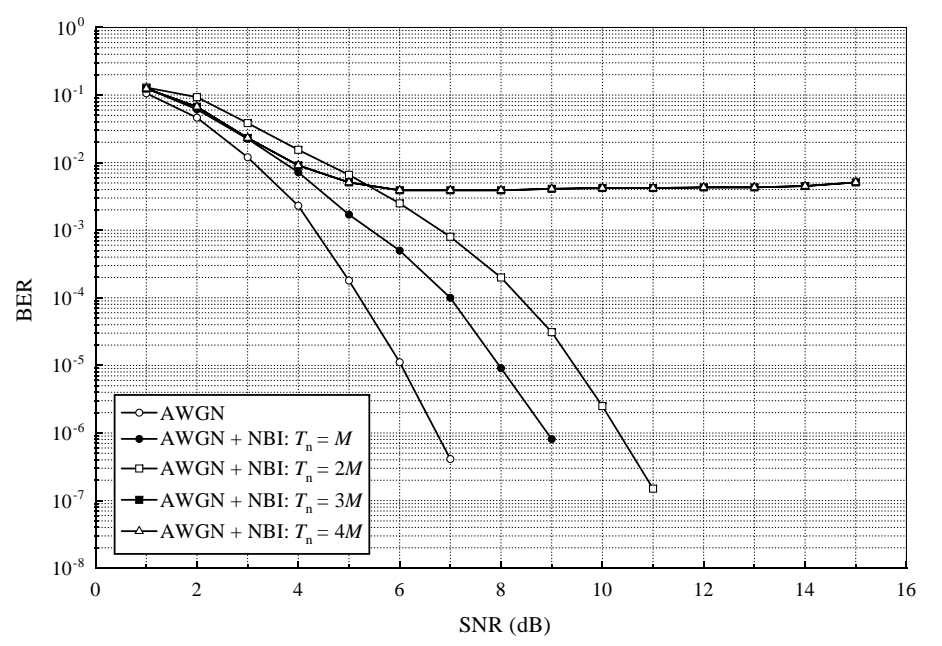

Figure 11. BER for AWGN with NBI at different values of $T_{n}$ and a probability of appearance of $\boldsymbol{p}_{n}=10^{-1}$.

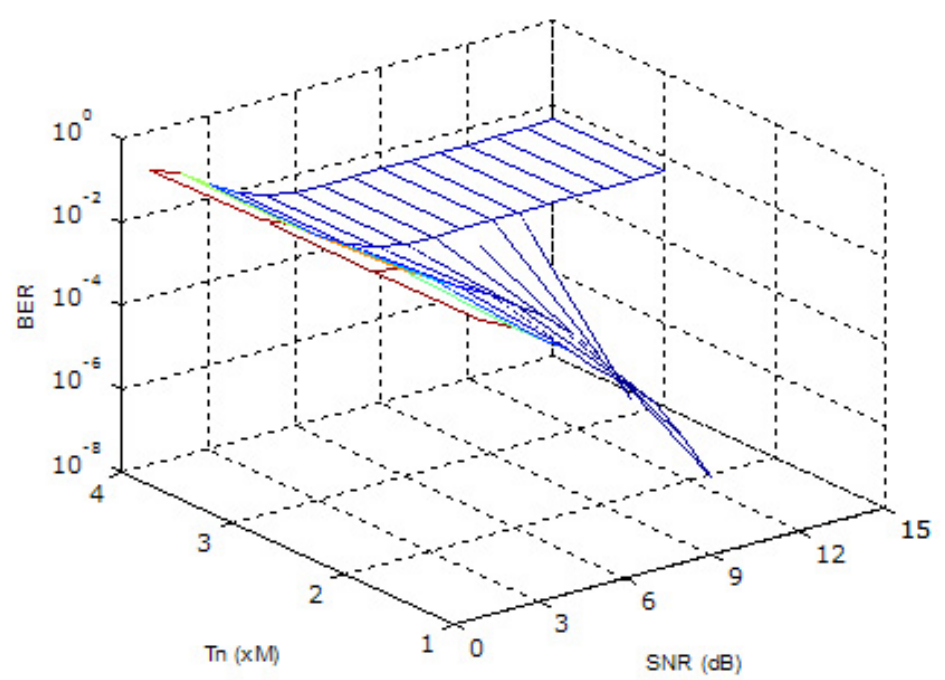

Figure 12. A 3-D graph of BER for AWGN with NBI at different values of $T_{n}$ and a probability of appearance of $\boldsymbol{p}_{n}=10^{-1}$.

We also run our simulations for different values of the probability of appearance $\boldsymbol{p}_{n}$ of the NBI for certain specific durations of time.

Figure 11 shows that the variation of the length or the duration of the NBI will affect badly the 


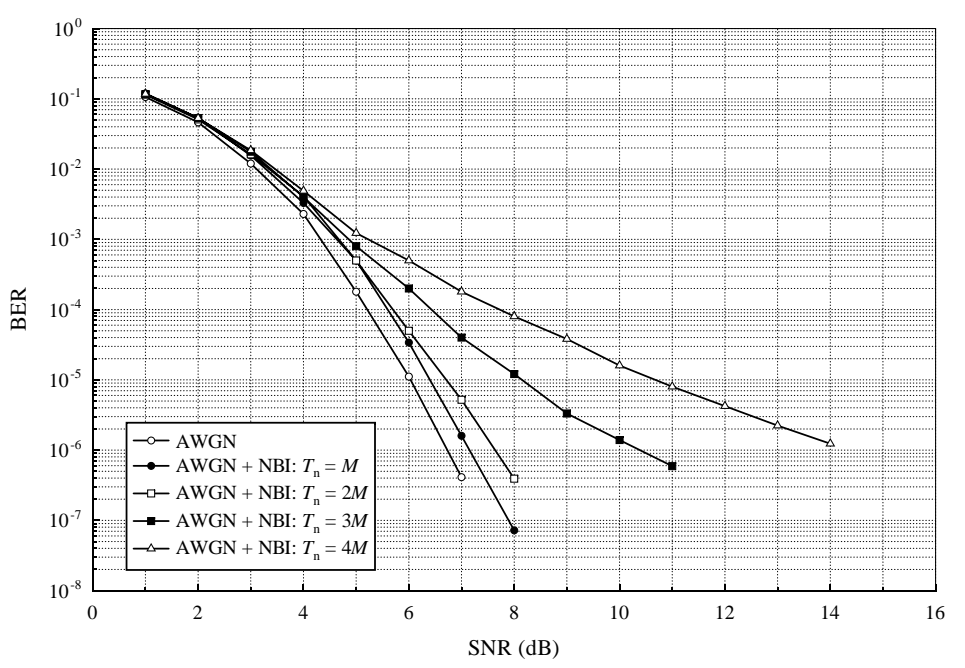

Figure 13. BER for AWGN with NBI at different values of $T_{n}$ and a probability of appearance of $\boldsymbol{p}_{n}=10^{-2}$.

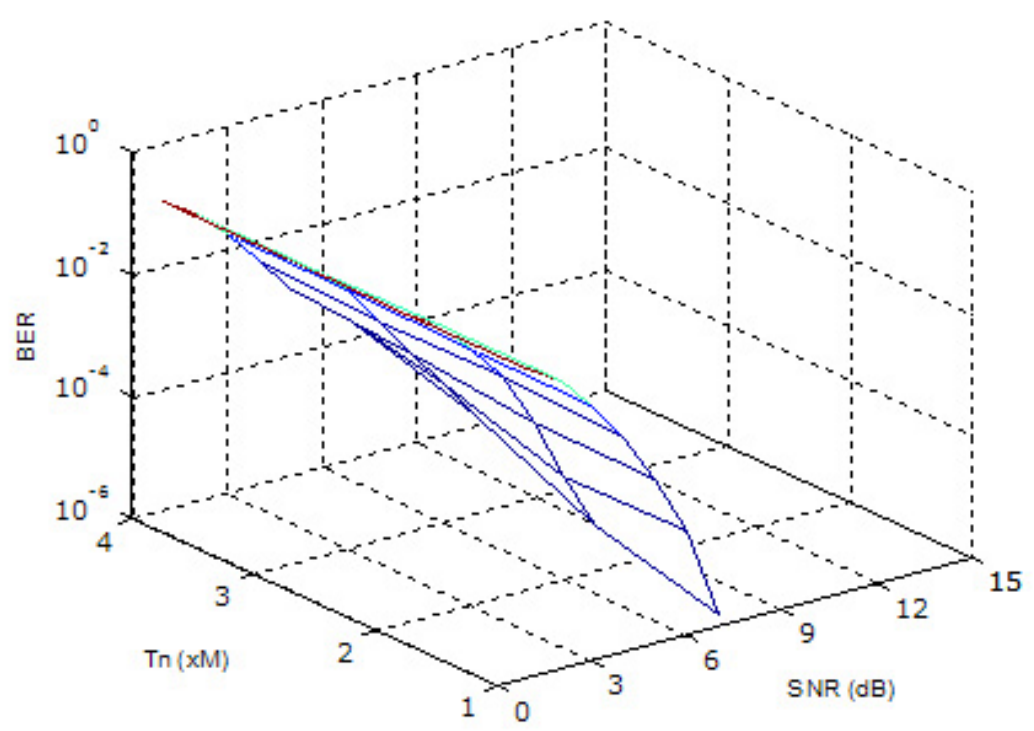

Figure 14. A 3-D graph of BER for AWGN with NBI at different values of $T_{n}$ and a probability of appearance of $\boldsymbol{p}_{n}=10^{-2}$.

performance of our code and the BER performance is deteriorating as much as $T_{n}$ is increasing. These results are taken for $\boldsymbol{p}_{n}=10^{-1}$.

Figure 12 is a three-dimensional representation of the BER in terms of $T_{n}$ and SNR per useful bit, which can help see the results from another perspective.

Figure 13 shows that the variation of the probability of appearance of NBI will affect badly the performance of our code and the BER performance is deteriorating as long as $T_{n}$ is increasing. These results are taken for $\boldsymbol{p}_{n}=10^{-2}$.

In a similar way, Figure 14 is a three-dimensional representation of the BER in terms of $\boldsymbol{p}_{n}$ and SNR, which can help see the results from another perspective. .

In Figure 15, we vary the probability of appearance $\boldsymbol{p}_{n}$ of NBI for a certain value of $T_{n}$, which is in this case $T_{n}=M$.

Figure 16 is a three-dimensional representation of the BER in terms of the probability of appearance $\boldsymbol{p}_{n}$ and SNR per useful bit, which can help see the results from another perspective and the case of 


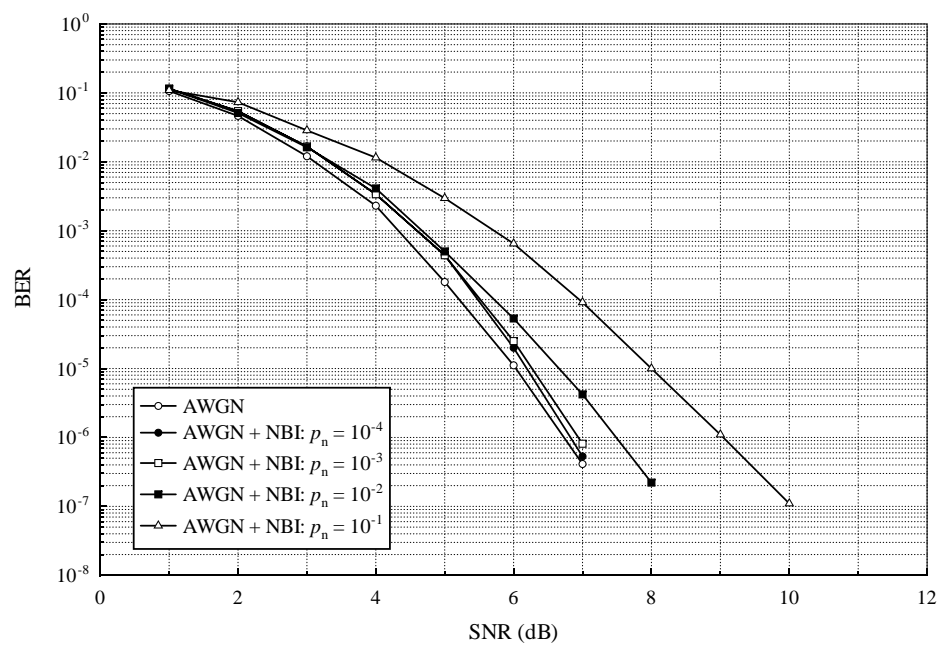

Figure 15. BER for AWGN with NBI at different values of $\boldsymbol{p}_{n}$ and a noise duration $T_{n}=M$.

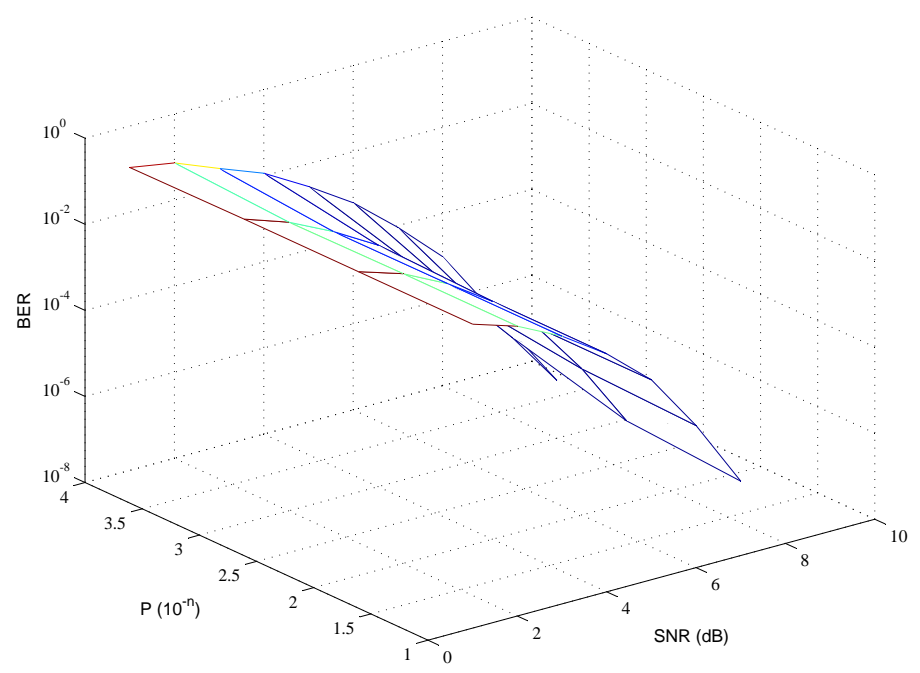

Figure 16. A 3-D graph of BER for AWGN with NBI at different values of $\boldsymbol{p}_{n}$ and a noise duration of $T_{n}=M$.

$T_{n}=M$.

Table 1. Gain at BER $=10^{-6}$ Compared to the BER for AWGN Only

\begin{tabular}{ccccc}
\hline \hline & \multicolumn{4}{c}{ Using a Fixed Error Probability of the NBI and Varied $T_{n}$} \\
\cline { 2 - 5 } NBI Probability & $T_{n}=M$ & $T_{n}=2 M$ & $T_{n}=3 M$ & $T_{n}=4 M$ \\
\hline $\boldsymbol{p}_{n}=10^{-1}$ & 1.7 & 2.2 & 3.4 & $\infty$ \\
$\boldsymbol{p}_{n}=10^{-2}$ & 0.3 & 0.8 & 2.4 & $>7.3$ \\
\hline \hline
\end{tabular}

In Figure 17, we vary the probability of appearance $\boldsymbol{p}_{n}$ of NBI for a certain value of $T_{n}$, which is in this case $T_{n}=2 \times M$. 


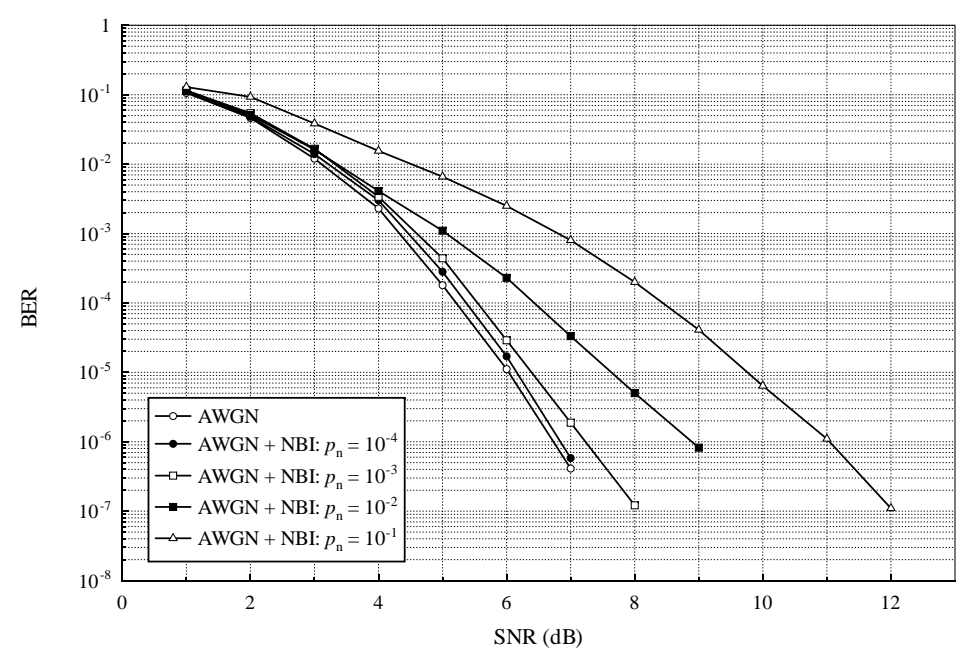

Figure 17. BER for AWGN with NBI at different values of $\boldsymbol{p}_{n}$ and a noise duration $T_{n}=2 \times M$.

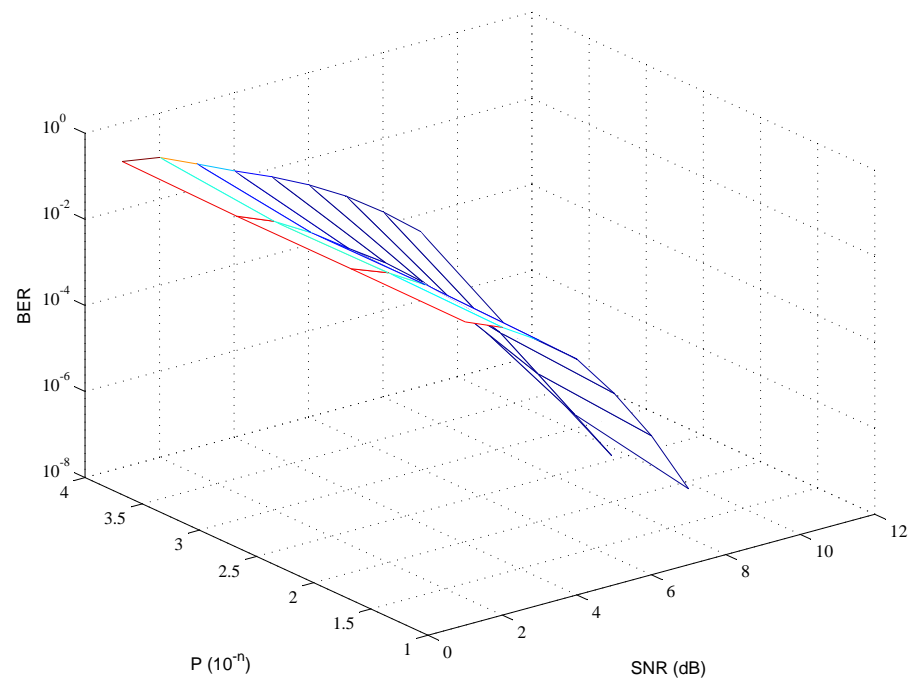

Figure 18. A 3-D graph of BER for AWGN with NBI at different values of $\boldsymbol{p}_{n}$ and a noise duration of $T_{n}=2 \times M$.

Table 2. Gain at BER $=10^{-6}$ Compared to the BER for AWGN Only

\begin{tabular}{ccccc}
\hline \hline & \multicolumn{4}{c}{ Using a Fixed Duration of the NBI and Varied $\boldsymbol{p}_{n}$} \\
\cline { 2 - 5 } NBI Duration & $\boldsymbol{p}_{n}=10^{-1}$ & $\boldsymbol{p}_{n}=10^{-2}$ & $\boldsymbol{p}_{n}=10^{-3}$ & $\boldsymbol{p}_{n}=10^{-4}$ \\
\hline$T_{n}=M$ & 2.2 & 0.7 & 0.2 & 0.08 \\
$T_{n}=2 M$ & 4.4 & 2.2 & 0.4 & 0.11 \\
\hline \hline
\end{tabular}

Figure 18 is a three-dimensional representation of the BER in terms of the probability of appearance $\boldsymbol{p}_{n}$ and SNR per useful bit, which can help see the results from another perspective and the case of $T_{n}=2 \times M$.

We can look at our results from another angel, where we see what is the fastest gain loss. And was is it caused by the increase of error probability of by the length of the NBI appearance or not? To do so , 
we compared our results to that obtained for the AWGN channel alone.

Table 1, which summarizes the results in Figures 11 and 13, shows the difference of gain loss when we vary only the length of the NBI appearance. Table 2, which summarizes the results in Figures 15 and 17, shows the difference of gain loss when we vary only the error probability of the NBI appearance. We can see from Tables 1 and 2, that the gain loss is more severe and increase dramatically under the effect of the length and the duration of the appearance of the narrow-band noise and not under the probability of its appearance.

\section{Conclusion}

In this paper we have introduced a new mathematical approach and implementation of combined permutation sequences and $M$-FSK modulation scheme. We have used the concept of permutation trellis codes to map permutation symbols to orthogonal frequencies for power line communications channel. This concept synchronizes the position of frequencies to the position of permutation symbols in a permutation sequence. The $M$-FSK modulation scheme has shown its robustness in a hostile environment as the power line channel in the presence of permanent frequency disturbances which are also called narrow band interferences.

We also made use of Walsh-Hadamard transforms and benefiting from the sign changes property in their matrices to replace permutation trellis codes for the same application's goal when implemented in harsh communications environments as the case for PLC. In this proposed mapping technique, we have chosen the corresponding $M$-FSK frequencies sequences for the corresponding transmitted signal, by using the properties of the Walsh-Hadamard transform.

The obtained results show the possibility of transmitting frequencies by corresponding permutation sequences or making used of sign changes using Walsh-Hadamard transform, could be a very good technique in combating narrowband interference in PLC channel.

Since our results are promising and for future work we will generalize our frequency mappings by the use of higher order of the Walsh-Hadamard transform and this can be with the use of convolutional codes with higher rates that can take our mapping to values higher than $M=4$ as for the case of $M=8,16,32, \ldots$ 


\section{Appendix}

Example 6.1. In the case of $M=8$, and after the stage of the multiplexer, we take an example of the transmitted frequency sequence as $\left\{f_{1}, f_{4}, f_{2}, f_{3}, f_{6}, f_{7}, f_{0}, f_{5}\right\}$. In the absence of noise, the output of the demodulator should look like

$$
\begin{aligned}
& t_{0} t_{1} t_{2} t_{3} t_{4} t_{5} t_{6} t_{7} \\
& \mathcal{F}=\begin{array}{l}
f_{0} \\
f_{1} \\
f_{2} \\
f_{3} \\
f_{4} \\
f_{5} \\
f_{5} \\
f_{6} \\
f_{7}
\end{array}\left[\begin{array}{lllllllllllll}
0 & 0 & 0 & 0 & 0 & 0 & 1 & 0 & 0 & 0 & 0 & 0 \\
0 & 0 & 0 & 1 & 0 & 0 & 0 & 0 \\
0 & 1 & 0 & 0 & 0 & 0 & 0 & 0 & 0 & 0 & 0 \\
0 & 0 & 0 & 0 & 1 & 0 & 0 & 0 \\
0 & 0 & 0 & 0 & 0 & 1 & 0 & 0
\end{array}\right]
\end{aligned}
$$

Channel noise causes errors, presented in boxes, in the received matrices, can be represented by $\mathcal{F}^{\prime}$ as depicted the following matrices for each type of noise.

- Background noise: Or the AWGN, the errors are reversal, where a one can be inverted to a zero or a zero can be inverted to a one.

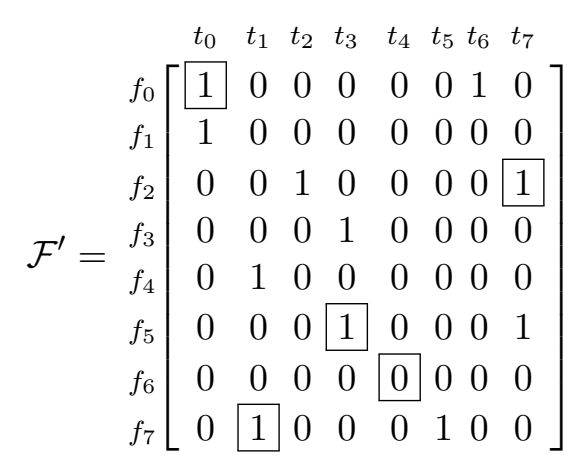

- Impulse noise: A total column in the matrix will be affected, where the received values are ones.

$$
\begin{aligned}
& t_{0} t_{1} t_{2} t_{3} t_{4} t_{5} \quad t_{6} t_{7}
\end{aligned}
$$

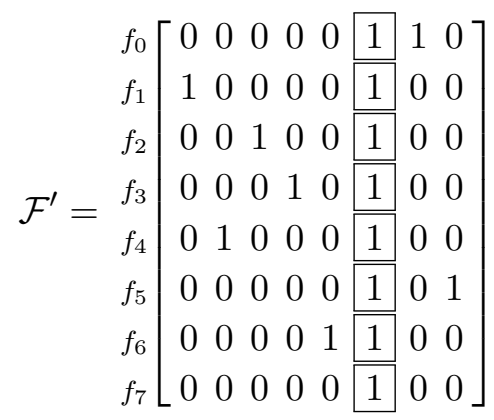

- Narrow band noise: A total row in the matrix will be affected, where the received values are ones.

$$
\begin{aligned}
& \begin{array}{llllllll}
t_{0} & t_{1} & t_{2} & t_{3} & t_{4} & t_{5} & t_{6} & t_{7}
\end{array} \\
& \mathcal{F}^{\prime}=\begin{array}{l}
f_{0} \\
f_{1} \\
f_{2} \\
f_{3} \\
f_{4} \\
f_{5} \\
f_{6} \\
f_{7}
\end{array}\left[\begin{array}{ccccccccc}
0 & 0 & 0 & 0 & 0 & 0 & 1 & 0 \\
1 & 0 & 0 & 0 & 0 & 0 & 0 & 0 \\
0 & 0 & 1 & 0 & 0 & 0 & 0 & 0 \\
0 & 0 & 0 & 1 & 0 & 0 & 0 & 0 \\
0 & 1 & 0 & 0 & 0 & 0 & 0 & 0 \\
0 & 0 & 0 & 0 & 0 & 0 & 0 & 1 \\
1 & 1 & 1 & 1 & 1 & 1 & 1 & 1 \\
0 & 0 & 0 & 0 & 0 & 1 & 0 & 0
\end{array}\right]
\end{aligned}
$$




\section{Example 6.2.}

Taking the case of $M=4$ and applying Equations (3) and (4) for the mapping of $\{00,01,10,11\} \rightarrow$ $\{1243,1342,4213,4312\}$, the distance metrics matrices of each set and their corresponding summations on the Hamming distances could be presented as follow,

$$
\begin{aligned}
& 00011011 \\
& \mathbf{D}=\begin{array}{l}
00 \\
01 \\
10 \\
11
\end{array}\left[\begin{array}{llll}
0 & 1 & 1 & 2 \\
1 & 0 & 2 & 1 \\
1 & 2 & 0 & 1 \\
2 & 1 & 1 & 0
\end{array}\right] \Rightarrow\|\mathbf{D}\|=16 \\
& 1243134242134312 \\
& \mathbf{E}=\begin{array}{l}
1243 \\
1342 \\
4213 \\
4312
\end{array}\left[\begin{array}{llll}
0 & 2 & 2 & 4 \\
2 & 0 & 4 & 2 \\
2 & 4 & 0 & 2 \\
4 & 2 & 2 & 0
\end{array}\right] \Rightarrow\|\mathbf{E}\|=32
\end{aligned}
$$

Taking into consideration the fact that the entries on the main diagonals are all zeros, we have $e_{i j}=d_{i j}+\delta$, with $\delta \geq 1$ for all $i \neq j$. The mapping of the outputs of the base code $\{00,01,10,11\}$ to the permutation set $\{1243,1342,4213,4312\}$, guarantees an increase of at least one unit of distance per step between any two unmerged paths in the trellis diagram of the resulting permutation trellis code, when comparing it to the base code.

Example 6.3. The flow chart presented in Figure 19 gives a detailed idea about all steps of mappings, from mapping binary sequences onto permutation sequences to the mapping of frequencies. The choice of the distance construction that provides the algorithm for the mapping is also taken into consideration in the low chart as it is a very important part in the mapping process.

The following mapping algorithm represents the code used for simulation to generate distance conserving mapping (DCM)for the case of $M=n=4$. The permutation symbols are represented by $y_{j}, j \leq M$ and the convolutional outputs, the binary sequences, are represented by $x_{i}, i \leq n$.

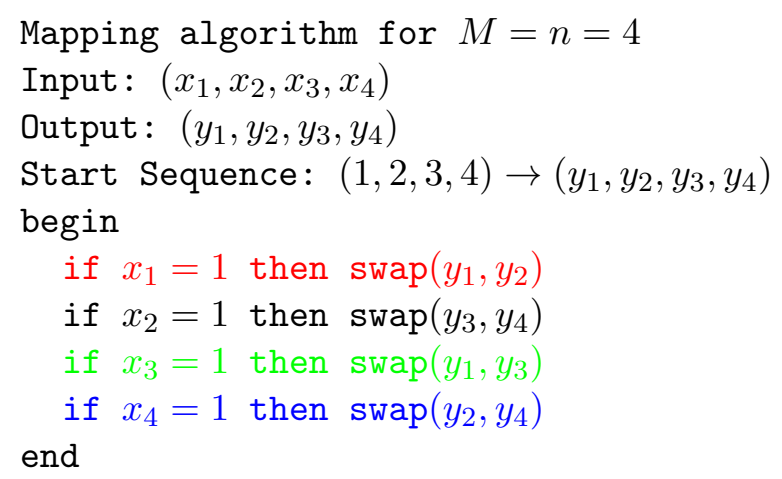

If we take the example of mapping $\{1,0,1,1\}$ onto an optimum permutation sequence, we can present the steps based on the above algorithm that generate for us the permutation symbols that will be substituting our binary sequences. We usually start with a random permutation sequences as the case of $\{1,2,3,4\}$, then following the prompts in the algorithm we reach at the end to the optimum permutation sequence, which is in this case $\{4,1,2,3\}$.

$$
\begin{aligned}
& 0000 \rightarrow \text { mapping } \rightarrow 1234 \\
& 1011 \rightarrow \operatorname{mapping} \rightarrow ? ? ? ? \\
& x_{1}=1 \rightarrow \operatorname{swap}\left(y_{1}, y_{2}\right) \rightarrow 2134 \\
& x_{2}=0 \rightarrow \operatorname{no} \operatorname{swap} \rightarrow 2134 \\
& x_{3}=1 \rightarrow \operatorname{swap}\left(y_{1}, y_{3}\right) \rightarrow 3124 \\
& x_{4}=1 \rightarrow \operatorname{swap}\left(y_{2}, y_{4}\right) \rightarrow 4123
\end{aligned}
$$




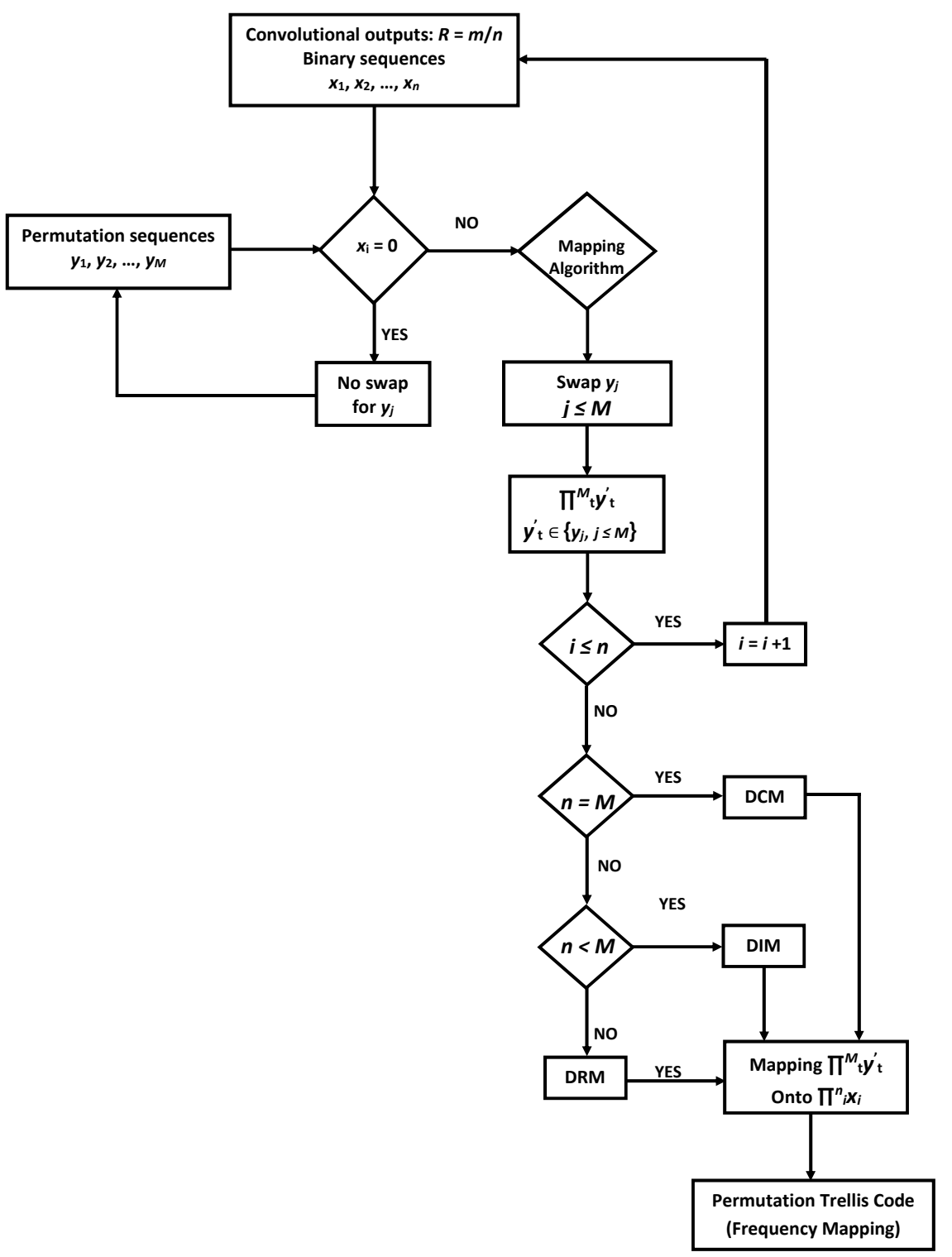

Figure 19. Flow Chart of the Frequency Mappings Process

Example 6.4. For $n=2$ and $n=3$, we have:

$$
H_{2}=\frac{1}{2}\left[\begin{array}{l}
+1+1+1+1 \\
+1-1+1-1 \\
+1+1-1-1 \\
+1-1-1+1
\end{array}\right]
$$




$$
H_{3}=\frac{1}{2 \sqrt{2}}\left[\begin{array}{l}
+1+1+1+1+1+1+1+1 \\
+1-1+1-1+1-1+1-1 \\
+1+1-1-1+1+1-1-1 \\
+1-1-1+1+1-1-1+1 \\
+1+1+1+1-1-1-1-1 \\
+1-1+1-1-1+1-1+1 \\
+1+1-1-1-1-1+1+1 \\
+1-1-1+1-1+1+1-1
\end{array}\right] \text {. }
$$

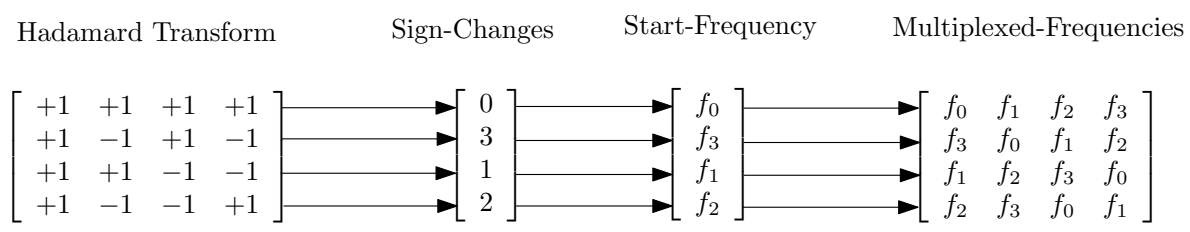

Figure 20. Hadamard matrix and frequency mapping: $M=4$

\section{Example 6.5.}

We take the example of $n=2$, where the order of the Hadamard matrix is 4 . Thus a 4-FSK modulator should be used for such application. Figure 20 shows that the generated number of sign changes from our Hadamard matrix is presented in the column in the form of the sequence $\{0,3,1,2\}$. Each number in this column or as we can call it symbol is in fact corresponding to a frequency with a proportional value to the symbol. These frequencies will be considered as the start frequencies for the 4-FSK sequences. Using the sign detector, which can be a logic circuit that can activate the switches which are linked to the signal generators, a 4-FSK signals is obtained by starting from the first frequency corresponding the first closing switch and followed by the rest of frequencies related to the number of sign changes. The 4-FSK transmitted signal is represented by the generated frequencies as depicted in Figure 20.

\section{References}

1. H. C. Ferreira, L. Lampe, J. Newbury, and T. G. Swart, Power Line Communications: Theory and Applications for Narrowband and Broadband Communications over Power Lines. Wiley, 2011.

2. A. J. H. Vinck, J. Haering, and T. Wadayama, Coded $m$-fsk for power line communications, in Proceedings of the IEEE International Symposium on Information Theory, p. 137, Sorrento, Italy, Jun. 25-30, 2000.

3. T. M. Lukusa, K. Ouahada, and H. C. Ferreira, Advantage of using permutation trellis codes and $m$ fsk modulation for power-line communications channel, in Proceedings of 2011 IEEE Africon, pp. 1-6, Livingstone, Zambia, Sept. 13-15, 2011.

4. J. Seberry, Orthogonal Designs: Hadamard Matrices, Quadratic Forms and Algebras. Springer, 2017.

5. T. M. Lukusa, K. Ouahada, and H. C. Ferreira, Frequency mappings with hadamard transform for power line communications channel, in Proceedings of the International Symposium on Power-Line Communications and its Applications, pp. 418-423, Udine, Italy, Apr. 3-6, 2011.

6. A. K. Mandal, Full-Optical TOAD based Walsh-Hadamard code generation. Springer, 2017.

7. P. Zheng and J. Huang, Efficient encrypted images filtering and transform coding with walshhadamard transform and parallelization, in Proc. IEEE Transactions on Image Processing, pp. 2541$2556,2018$.

8. W. Chen, L. Wang, Y. Fan, H. Lin, and X. Wei, Efficient resource allocation and interference management using compressive sensing in dense mobile communication systems, in 2016 8th International Conference on Wireless Communications \& Signal Processing (WCSP), pp. 1-6, 2016. 
9. http://www.cenelec.eu/Cenelec/Homepage.htm.

10. L. Lampe, A. M. Tonello, and T. G. Swart, Power Line Communications: Principles, Standards and Applications from Multimedia to Smart Grid. Wiley, 2016.

11. A. J. H. Vinck and J. Haering, Coding and modulation for power line communications, in Proceedings of the International Symposium on Power-Line Communications and its Applications, pp. 265-272, Limerick, Ireland, 2000.

12. S. Haykin, Communication Systems. John Wiley \& Sons, Inc., New York, 2009.

13. A. Viterbi and J. Omura, Principles of Digital Communication and Coding. Dover Publications, USA, 2013.

14. R. El-Bardan, E. Masazade, O. Ozdemir, Y. S. Han, and P. K. Varshney, Permutation trellis coded multi-level fsk signaling to mitigate primary user interference in cognitive radio networks, IEEE Trans. Commun., vol. 64, no. 1, pp. 104-11, 2016.

15. K. Ouahada and H. C. Ferreira, $k$-cube construction mappings from binary vectors to permutation sequences, in Proceedings of the IEEE International Symposium on Information Theory, pp. 630-634, Seoul, South Korea, June 28-July 3, 2009. 